ORIGINAL ARTICLE

\title{
Non-specific multiple ulcers of the small intestine unrelated to non-steroidal anti-inflammatory drugs
}

\author{
T Matsumoto, M lida, T Matsui, T Yao, H Watanabe, T Yao, H Okabe
}

J Clin Pathol 2004;57:1145-1150. doi: 10.1136/icp.2003.015735

See end of article for authors' affiliations ....................

Correspondence to: Dr T Matsumoto, Department of Medicine and Clinical Science, Graduate School of Medical Sciences, Kyushu University, Maidashi 3-1-1, Higashi-ku, Fukuoka 812-8582 Japan; matane@intmed2. med.kyushu-u.ac.jp

Accepted for publication 25 March 2004

\begin{abstract}
Aim: Non-steroidal anti-inflammatory drug (NSAID) enteropathy is a cause of chronic blood and protein loss from the intestine. The aim of this investigation was to compare NSAID enteropathy with enteropathy not related to chronic NSAID use.

Patients/Methods: During the period 1967-97, 12 cases of non-specific small intestinal multiple ulcers were laparotomised because of chronic and persistent blood loss for a prolonged period. The clinical features before and after surgery, and the small intestinal lesions, were compared between NSAID users and non-users.

Results: Three patients were NSAID users and the other nine were not. Initial clinical manifestations, haemoglobin concentrations, serum protein values, and inflammatory reactions were similar in the two groups. In both groups there were multicentric stenoses in the small intestine. In those not using NSAIDs, the stenoses were accompanied by multiple, sharply demarcated small ulcers with minimal and nonspecific chronic inflammatory infiltrates. The small intestinal ulcers in NSAID users showed linear and circumferential alignment. NSAID users were free from medication during the subsequent period of observation, whereas repeated laparotomy and medication were required in eight of the nine patients not using NSAIDs.

Conclusion: There appears to be a chronic enteropathy, not related to the use of NSAIDs, in which nonspecific multiple ulcers are found. This enteropathy may be a distinct entity that causes persistent blood and protein loss from the intestine.
\end{abstract}

"A proportion of non-specific small intestinal ulcers are caused by thiazides, potassium tablets, or non-steroidal anti-inflammatory drugs"

Previously, we anecdotally reported an enteropathy characterised by multiple small intestinal ulcers and chronic, persistent blood loss. ${ }^{13-15}$ The cases were characterised by multiple, sharply demarcated ulcers in the small intestine and chronic, refractory anaemia in adolescents. ${ }^{13-15}$ Because several small intestinal pathologies have subsequently been established as the cause of small intestinal ulcers, we thought that it would be interesting to reanalyse the histological and clinical features of these chronic small intestinal lesions. Thus, we retrospectively analysed the clinical course and histological findings of our subjects with non-specific small intestinal multiple ulcers, with the aim of proposing a clinicopathological entity distinct from NSAID enteropathy.

\section{MATERIALS AND METHODS \\ Subjects}

We reviewed the clinical files of the patients with small intestinal pathology diagnosed at our institution during the period 1967-97. Each case file contained all of the clinical records at the initial admission, small bowel radiography, and the pathological records of biopsy samples or surgically resected specimens. Among the files, we identified 160 patients who had undergone surgery because of small intestinal ulcer or stricture. A review of the clinical records of these patients identified 91 patients who had had symptoms for more than a year before surgery. One patient had an obvious history of abdominal irradiation, and five had extraintestinal symptoms compatible with a diagnosis of Behcet's disease. ${ }^{16}$ Among the other 85 patients, 37 were lost to follow up after surgery. We excluded these 43 patients. In 48 patients, the clinical data at the time of initial surgery and subsequent clinical course at our institutions until April 2002 could be verified.

Thirteen subjects satisfied the criteria for Crohn's disease (see below), and eight satisfied those of intestinal tuberculosis. In two subjects, one or more clinical items for each disease turned out to be positive during the subsequent

Abbreviations: CMUSE, cryptogenic multifocal ulcerous stenosing enteritis; CRP, $C$ reactive protein; NSAID, non-steroidal antiinflammatory drugs 
Table 1 Comparison of clinical features at diagnosis between NSAID users and non-users

\begin{tabular}{lll} 
& Non-users $(\mathbf{n}=9)$ & NSAID users $(\mathbf{n}=3)$ \\
\hline Sex (female/male) & $7 / 2$ & $0 / 3$ \\
Age at onset (years)* & $15,12-53$ & $68,55-88$ \\
Presenting symptom & & \\
Abdominal pain & 2 & 2 \\
Diarrhoea & 0 & 0 \\
Haematochezia & 1 & 0 \\
Anaemia & 8 & 3 \\
Emaciation & 8 & 3 \\
Age at diagnosis (years)* & $27,13-58$ & $70,57-88$ \\
Drug exposure & 0 & 3 \\
Diclofenac & 0 & 1 \\
Loxoprofen & 0 & 2 \\
Indomethacin & 0 & 1 \\
Thiazide & 0 & 0 \\
Potassium tablet & 0 & 0 \\
Underlying condition & & \\
Absent & 9 & 0 \\
Osteoarthritis & 0 & 3 \\
Haemoglobin (g/l)* & $75,62-88$ & $58,48-82$ \\
Serum protein (g/l)* & $49,46-69$ & $59,48-69$ \\
Positive C reactive protein & 1 (7.7\%) & $1(16.7 \%)$ \\
\hline *Values are expressed as median and range. & \\
NSAID, non-steroidal anti-inflammatory drug. & \\
\hline & &
\end{tabular}

clinical course (one for Crohn's disease and the other for intestinal tuberculosis). In another three patients, the criteria could not be fully assessed. In 10 subjects, information of possible NSAID use before surgery could not be verified. We excluded these 36 patients from further analyses. The remaining 12 patients were the subjects of our present investigation.

\section{Exclusion criteria}

\section{Crohn's disease}

The diagnosis of Crohn's disease was established according to the Japanese criteria proposed by one of the authors. ${ }^{17}$ The small intestinal lesions characterised by longitudinal ulcers and cobblestone appearance were the first diagnostic criteria for Crohn's disease. Histological confirmation of non-caseating granuloma in the endoscopically obtained biopsy specimen anywhere within the gastrointestinal tract was regarded to be the second diagnostic criteria for Crohn's disease. Subjects who satisfied the first criteria or those with small intestinal ulcers who satisfied the second criteria were diagnosed as having Crohn's disease.

Intestinal tuberculosis

Confirmation of mycobacterium species in the faeces or in the biopsy specimens was the gold standard for the diagnosis of intestinal tuberculosis. Histologically verified caseating epithelioid granuloma in the resected specimen was the second criteria for the diagnosis. Furthermore, improvement of small intestinal lesions after antimycobacterial agents was the third diagnostic criteria. Subjects who had any one of the three criteria were diagnosed as having intestinal tuberculosis.

\section{Definition of chronic NSAID use}

All of the prescribed medications before surgery were identified by a review of clinical charts at the time of initial surgery. Intake of the medications was confirmed by direct contact with the subjects at the latest visit to our institutions. We also questioned the subjects about possible intake of commercially available medications. Chronic NSAID use was defined as a continuous prescription of NSAID for more than
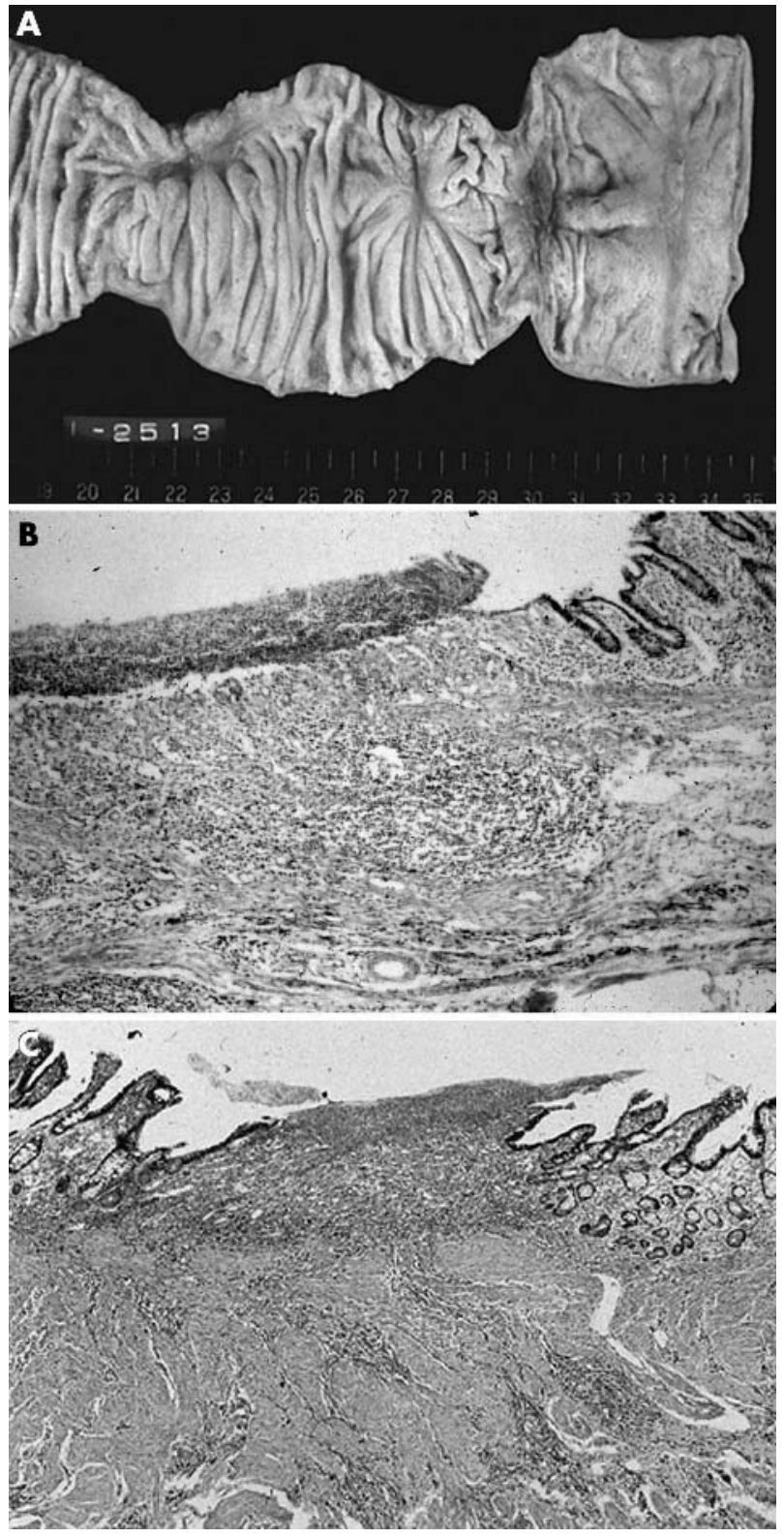

Figure 1 Small intestinal ulcers in a patient not using non-steroidal antiinflammatory drugs. (A) There are two ulcers with fold convergencies in the ileum. The intervening mucosa is intact. (B) A low power view of the affected ileum reveals the ulcer to be restricted to the submucosal layer with fibrosis. (C) A high power view demonstrates a mucosal defect with mild fibrosis and minimal inflammatory infiltrates.

a month before surgery. Other short term use was not counted as chronic NSAID use.

\section{Pathological assessment}

The surgically removed small intestinal specimens were reviewed by two pathologists (HW and TY) who were blind to the clinical diagnosis. The resected specimens were examined macroscopically with regard to the number and the shape of the ulcers. The alignment of the ulcers was classified as longitudinal, circular, or unspecified. The histological variables were examined in the slices cut from the centre of the ulcers, which were stained with haematoxylin and eosin. The histological variables examined were villous atrophy and inflammatory infiltrates in the mucosa, in addition to depth of ulcer, oedema, fibrosis, and inflammatory infiltrates. 


\section{Clinical features and outcome}

Based on the clinical records at the initial admission, presenting symptoms, previous medications, haemoglobin, $C$ reactive protein (CRP), serum protein values, and prescriptions at the time of surgery were reviewed. The clinical outcome after surgery was assessed with respect to repeated laparotomy and extraintestinal manifestations during the observation period and to clinical parameters at the final visit.

\section{RESULTS}

At the time of initial surgery, three subjects had a history of chronic NSAID use. The prescribed NSAIDs included diclofenac, loxoprofen, and indomethacin. The duration of the NSAID use ranged from three to 16 years. These subjects were regarded to be NSAID users. The other nine subjects had not been prescribed NSAIDs before surgery, and they denied commercially available drug exposure. These nine subjects were categorised as non-users of NSAIDs.

\section{Comparison of initial clinical features}

Table l compares the clinical features of the NSAID users and non-users. The age at onset was greater in NSAID users (median age, 57; range, 24-86 years) than in non-users (median, 15; range, 12-53 years). Similarly, NSAID users were younger at the time of diagnosis (median, 27; range, 1358 years) than were non-users (median, 70; range, 57-88 years). In both NSAID users and non-users, anaemia was the most frequent presenting symptom. Two NSAID users and two non-users complained of abdominal pain. In NSAID users, NSAID was indicated for osteoarthritis. There was no underlying disorder in non-NSAID users. None of the patients had been given thiazide or potassium tablets.

Comparisons of laboratory data showed that both NSAID users and non-users had low concentrations of haemoglobin and serum protein. The median (range) haemoglobin (75, 62-88 g/l $v$ 58, 48-82 g/l) and serum protein values $(49,46-$ $69 \mathrm{~g} / \mathrm{l} v$ 59, 48-69 g/l) were similar in non-NSAID users and NSAID users, respectively. CRP was positive in two subjects (one in each group).

\section{Comparison of small intestinal lesions}

The intestinal ulcers in both NSAID users and non-users were found predominantly in the ileum. Table 2 compares the macroscopic findings in the small intestinal lesions between the two groups. The intestinal ulcers in non-NSAID users were characterised by multiple, sharply demarcated mucosal defects (fig 1A). The ulcers had various configurations, referred to as irregular shape or circular shape. In six nonNSAID users, the ulcers were located predominantly on one

Table 2 Comparison of macroscopic findings between NSAID users and non-users

\begin{tabular}{lll}
\hline & $\begin{array}{l}\text { Non-NSAID users } \\
(\mathbf{n}=9)\end{array}$ & $\begin{array}{l}\text { NSAID users } \\
(\mathbf{n}=3)\end{array}$ \\
\hline $\begin{array}{l}\text { Number of ulcers (median } \\
\text { (range)) }\end{array}$ & $6(3-12)$ & $10(8-11)$ \\
$\begin{array}{l}\text { Longitudinal alignment } \\
\text { Mesenteric }\end{array}$ & 3 & 0 \\
$\quad$ Anti-mesenteric & 3 & 0 \\
$\quad$ No eccentricity & 3 & 3 \\
$\begin{array}{l}\text { Predominant shape of ulcers } \\
\text { Irregular }\end{array}$ & 6 & 0 \\
Longitudinal & 0 & 0 \\
$\quad$ Circular & 3 & 3 \\
Margin of ulcer & 9 & 3 \\
$\quad$ Clear & 0 & 0 \\
Irregular & 0 & \\
\hline NSAID, non-steroidal anti-inflammatory drug. &
\end{tabular}

side (the mesenteric side in three subjects and antimesenteric side in three subjects). In contrast to non-NSAID users, the small intestinal ulcers in NSAID users showed uniform macroscopic findings. The ulcers in these subjects were characterised by circular and linear mucosal defects (fig 2A). In both NSAID users and non-NSAID users, the intervening mucosa appeared to be normal.

\section{Comparison of histological findings}

Table 3 compares the histological findings in the small intestine between the two groups. In both groups, the maximal depth of ulcer in the resected small intestine was

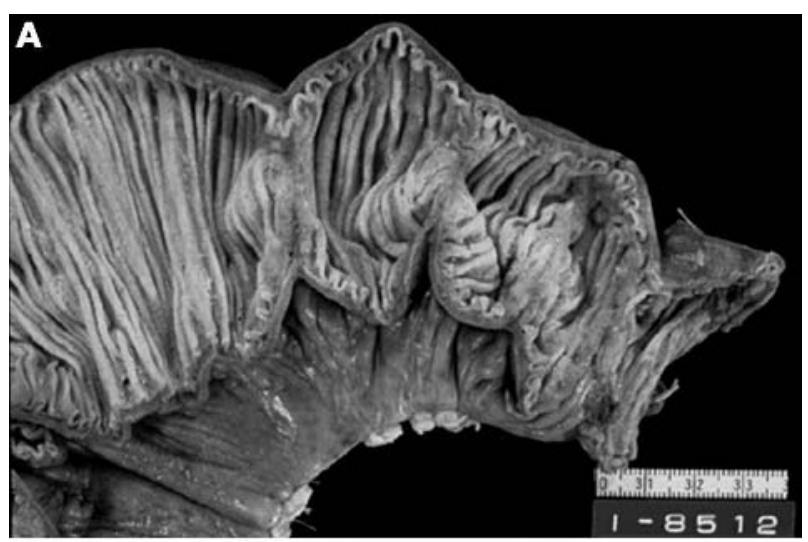

B

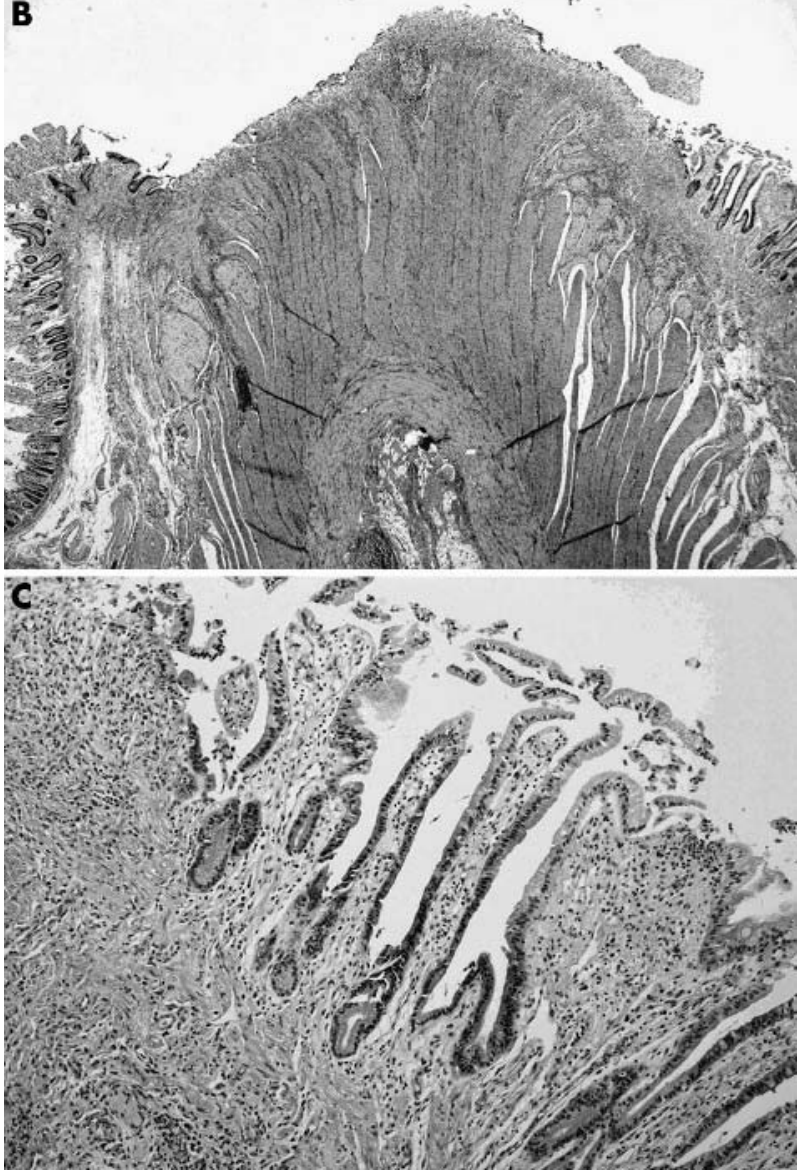

Figure 2 Small intestinal ulcers in a non-steroidal anti-inflammatory drug user. (A) There are two linear and circumferential ulcers in the ileum. (B) Microscopically, the ulcer is restricted to the submucosal layer with submucosal fibrosis. (C) A high power view of the ulcer shows fibrosis with mild inflammatory infiltrates. 
the submucosal layer (figs 1B, 2B). Submucosal oedema was not evident, except for in one non-NSAID user. There was mild fibrosis in the submucosal layer in all three NSAID users and in eight of the nine non-users (figs 1C, 2C). Although a mild chronic inflammatory infiltrate was found in six subjects in the non-NSAID group, it was not seen in the NSAID users. In both groups, mucosal atrophy was not seen in the intervening mucosa. There were no cases of vasculitis or lymphangitis in the two groups.

\section{Comparison of clinical course}

Table 4 compares the follow up data between the two groups. The follow up period after the initial laparotomy ranged from 2 to 35 years (median, 22) in non-NSAID users and from 2.0 to 4.5 years (median, 3 years) in NSAID users. Both NSAID users and non-NSAID users were free from continuous NSAID intake during the observation period. Whereas NSAID users were free from medications, eight of the nine nonNSAID users were taking oral or intravenous iron supplements. In addition, five non-NSAID users were treated by enteral or parenteral nutrition.

Laboratory data at the final visit were not available in two non-NSAID users. In the remaining five non-NSAIDs users, median (range) haemoglobin values (111, 86-139 g/l) and serum protein values $(64,51-74 \mathrm{~g} / \mathrm{l})$ were lower than in NSAID users (haemoglobin: 145, 93-161 g/l and serum protein $74,66-78 \mathrm{~g} / \mathrm{l}$ ).

During the observation period, eight non-NSAID users underwent repeated laparotomy. In contrast, none of the NSAID users underwent laparotomy. A non-NSAID user died of liver cirrhosis 21 years after the diagnosis of small intestinal ulcers. The other patients were alive in April, 2002. In non-NSAID users, no autoimmune, haematological, or vascular disorders occurred during the observation period. An NSAID user was diagnosed as having myelodysplastic syndrome two years after the diagnosis of enteropathy.

\section{DISCUSSION}

The results of our retrospective case study show that a group of patients exists in whom chronic, non-specific small intestinal ulcers are present that cannot be attributed to NSAID use. A criticism of our study could be that the number of subjects is too small to draw strong conclusions and that our non-NSAID users may have actually been exposed to NSAIDs. However, the small number of the subjects was the consequence of the strict criteria applied, which were based

Table 3 Comparison of histological findings between NSAID users and non-NSAID users

\begin{tabular}{lll}
\hline & $\begin{array}{l}\text { Non-NSAID users } \\
(\mathbf{n}=9)\end{array}$ & $\begin{array}{l}\text { NSAID users } \\
(\mathbf{n}=3)\end{array}$ \\
\hline $\begin{array}{l}\text { Maximal depth of ulcer } \\
\quad \text { Submucosa }\end{array}$ & 9 & 3 \\
$\quad \begin{array}{l}\text { Proper muscular layer } \\
\text { Submucosal oedema }\end{array}$ & 0 & 0 \\
$\quad$ None & 8 & 3 \\
Mild & 1 & 0 \\
$\quad$ Moderate & 0 & 0 \\
Fibrosis & 1 & 0 \\
$\quad$ None & 8 & 3 \\
Mild & 0 & 0 \\
Moderate & 3 & 3 \\
Inflammatory infiltrate & & 0 \\
$\quad$ None & 0 & \\
Mild & 0 & 3 \\
Moderate & 0 \\
Intervening mucosa & & \\
$\quad$ Mucosal atrophy (-) & 9 & \\
Mucosal atrophy (+) & 0 & \\
\hline NSAID, non-steroidal anti-inflammatory drug. & \\
\hline
\end{tabular}

Table 4 Comparison of clinical course between nonNSAID users and NSAID users

\begin{tabular}{|c|c|c|}
\hline & $\begin{array}{l}\text { Non-NSAID users } \\
(n=9)\end{array}$ & $\begin{array}{l}\text { NSAID users } \\
(\mathrm{n}=3)\end{array}$ \\
\hline Observation period (years)* & $22,2-35$ & $3.5,2.0-4.5$ \\
\hline \multicolumn{3}{|l|}{ Repeated surgery } \\
\hline None & 1 & 3 \\
\hline Once & 4 & 0 \\
\hline Twice or more & 4 & 0 \\
\hline \multicolumn{3}{|l|}{ Medication for intestinal pathology } \\
\hline No & 1 & 3 \\
\hline Yes & 8 & 0 \\
\hline Iron supplementation & 7 & \\
\hline Enteral or parenteral nutrition & 5 & \\
\hline Mesalazine & 1 & \\
\hline \multicolumn{3}{|l|}{ Extraintestinal manifestations } \\
\hline None & 6 & 2 \\
\hline Autoimmune disorder & 0 & 0 \\
\hline Haematological disorder & 0 & 1 \\
\hline Vascular disorder & 0 & 0 \\
\hline Final clinical parameters* & $(n=7)$ & $(n=3)$ \\
\hline Haemoglobin (g/l) & $111,59-139$ & $116,93-161$ \\
\hline Serum protein (g/l) & $63,51-74$ & $67,58-78$ \\
\hline $\mathrm{C}$ reactive protein (g/l) & $2,1-4$ & $0,0-2$ \\
\hline \multicolumn{3}{|l|}{ Clinical outcome } \\
\hline Alive & 8 & 6 \\
\hline Deceased & 1 & 0 \\
\hline
\end{tabular}

on clinical data through initial surgery until the latest visit. We believe that small intestinal pathology of obscure origins and uncensored chronic NSAID use was excluded as much as is possible by such an approach, although exposure to a small amount of NSAIDs could not be completely excluded.

Over the past decade, it has become widely accepted that NSAIDs damage the small intestine. Bjarnason et al were the first to investigate longterm NSAID users who had small intestinal stricture and persistent anaemia. ${ }^{18}$ Their observations established the concept of NSAID enteropathy characterised by persistent blood loss from the small intestine. The macroscopic lesions in NSAID enteropathy consist of multiple circumferential ulcers with severe concentric stenosis, referred to as diaphragm disease. ${ }^{18-20}$ The macroscopic findings of the small intestinal lesions in our NSAID users were identical to those reported previously.

Diaphragm disease of the intestine has non-specific histology, such as fibrosis in the submucosa and thickening of the muscularis mucosae. ${ }^{18-22}$ The intestinal ulcers in our non-NSAID users were in fact non-specific, and they shared common characteristics with those found in NSAID users. In both groups of patients, the ulcers were confined to the mucosa or to the superficial submucosal layer and had mild fibrosis and minimal oedema, without pronounced inflammatory infiltrates.

Because non-specific histology was the only finding, diaphragm disease coupled with the use of NSAIDs is a pathognomic feature of NSAID enteropathy. ${ }^{18}{ }^{20}$ However, Santolaria et al recently reported a Spanish patient with diaphragm disease who had a 25 year history of relapsing abdominal pain and oedema. ${ }^{23}$ Similar to our non-NSAID users, this patient was not a chronic NSAID user. ${ }^{23}$ Thus, longterm NSAID use is probably not the only cause of diaphragm disease and chronic blood loss.

The initial manifestations of the disease in non-NSAID users were identical to those of NSAID users. Both groups of patients complained of persistent abdominal symptoms and, furthermore, they had pronounced anaemia and hypoproteinaemia. Even though the cause of the intestinal ulcers in our non-NSAID users remains obscure, and the lesions may be heterogeneous in nature, chronic NSAID abuse was the 
only clinical feature that could distinguish between the two entities. In contrast, the subsequent clinical course was very different between the two groups with respect to relapse and management of the disease.

Before the recognition of NSAID enteropathy there were some descriptions of non-specific small intestinal ulcers, particularly during the $1960 \mathrm{~s},{ }^{3-6}$ and thiazides or potassium tablets were presumed to have contributed to their pathogenesis. $^{72425}$ However, approximately $60 \%$ of the reported cases were thought to be unrelated to medications. ${ }^{26}$ Because NSAID compounds (except for acetylsalycilate) were not widely available at that time, it seems unlikely that the nonspecific small intestinal ulcers in the literature were caused by NSAIDs.

In 1979, Modigliani et al reported four patients with small intestinal multiple ulcers accompanied by villous atrophy, and they referred to the entity as chronic non-specific ulcerative duodenojejunoileitis. ${ }^{10}$ Robertson et al subsequently reported seven patients with similar clinicopathological features. ${ }^{11}$ Modigliani and colleagues and Robertson and colleagues ${ }^{11}$ suggested that the small intestinal ulcers might be related to coeliac disease, because their patients had been diagnosed with this disease or because the ulcers were accompanied by various degrees of mucosal atrophy. Similar cases have been reported in the literature with various nomenclatures, such as ulcerative jejunoileitis, ulcerative enteritis, and chronic non-specific ulcerative duodenojejunoileitis. ${ }^{27}$ Patients with this disease manifest with fever and diarrhoea, and their small intestine has non-specific inflammatory infiltrates and mucosal atrophy.

\section{"Whereas cryptogenic multifocal ulcerous stenosing enteritis is characterised by multiple, small intestinal stenoses, the ulcers in our non-NSAID users have variable macroscopic characteristics, and do not always result in stenoses"}

Another distinctive entity of non-specific small intestinal multiple ulcers has been described as CMUSE (cryptogenic multifocal ulcerous stenosing enteritis). ${ }^{12}$ Although descriptions of CMUSE are not very detailed and most cases have been reported in the French literature, ${ }^{28}$ Perlemuter et al recently analysed 12 patients with this disease. ${ }^{12}$ In their report, the clinicopathological features of CMUSE were summarised as: (1) unexplained small intestinal strictures found in adolescents and middle aged subjects, (2) superficial ulceration of the mucosa and submucosa, (3) chronic or relapsing clinical course even after surgery, (4) no biological signs of systemic inflammatory reaction, and (5) beneficial effect of steroids. Although a particular form of arteritis is presumed to be related to the pathogenesis of CMUSE, ${ }^{29}$ manifestations of vasculitis are not necessarily evident in all the cases. ${ }^{12}$

The intestinal lesions of our non-NSAID users seem to be slightly different from those of CMUSE. Whereas CMUSE is characterised by multiple, small intestinal stenoses, the ulcers in our non-NSAID users have variable macroscopic characteristics, and do not always result in stenoses. In addition, because none of our non-NSAID users was treated with steroids, the therapeutic effect of these drugs is not known. However, other manifestations and the clinical course of our non-NSAID users were compatible with those of CMUSE. In contrast, coeliac disease-which is a condition that is thought to predispose the patient to chronic nonspecific ulcerative duodenojejunoileitis or atrophy in the intervening normal mucosa-was not evident in our nonNSAID users. These observations suggest that the enteropathy of our non-NSAID users may be categorised as a
Take home messages

- A chronic enteropathy of non-specific small intestinal multiple ulcers appears to exist that is not related to the use of non-steroidal anti-inflammatory drugs (NSAIDs)

- This enteropathy may be a distinct entity that causes persistent blood and protein loss from the intestine

- A review of the literature indicated that this enteropathy shares common clinical features with the recently recognised cryptogenic multifocal ulcerous stenosing enteritis, and should be considered in patients with intestinal stricture who are not longterm NSAID users

variant of CMUSE, rather than chronic non-specific ulcerative duodenojejunoileitis.

In conclusion, our results indicate that there may be a chronic enteropathy of non-specific small intestinal multiple ulcers, which is distinct from NSAID enteropathy. In addition, the small intestinal multiple ulcers in our nonNSAID users do not seem to be a consequence of putative underlying conditions, because a prolonged period of observation failed to identify specific extraintestinal manifestations. A review of the literature indicated that the enteropathy shares common clinical features with the recently recognised CMUSE. Although this type of enteropathy seems to be rare, it should be seriously considered in subjects with intestinal stricture who are not longterm NSAID users.

\section{Authors' affiliations}

T Matsumoto, M lida, Departments of Medicine and Clinical Science, Graduate School of Medical Sciences, Kyushu University, Fukuoka 8128582, Japan

T Matsui, T Yao, Department of Gastroenterology, Fukuoka University Chikushi Hospital, Chikushino 818-8502, Japan

T Yao, Department of Anatomic Pathology, Graduate School of Medical Sciences, Kyushu University

H Watanabe, Division of Molecular and Diagnostic Pathology,

Department of Molecular Genetics, Course for Molecular and Cellular Medicine, Graduate School of Medical and Dental Sciences, Niigata University, Niigata 951-8501, Japan

H Okabe, Department of Internal Medicine, Kitasato University School of Medicine, Kanagawa 228-8555, Japan

\section{REFERENCES}

1 Lewis BS. Small intestinal bleeding. Gastroenterol Clin North Am 2000;29:67-95.

2 Caspary WF, Stein J. Diseases of the small intestine. Eur J Gastroenterol Hepatol 1999;11:21-5.

3 Graham DY, Bynum TE. Primary nonspecific small bowel ulceration as a source of chronic bleeding. Report of a case and review of the approach to localization of the site of small bowel hemorrhage. Am J Gastroenterol 1974;62:350-5.

4 Glynn MJ, Pendower J, Shousha S, et al. Recurrent bleeding from idiopathic ulceration of small bowel. BMJ 1984;288:975-6.

5 Wilson IR, Cooley NV, Luibel FJ. Nonspecific stenosing small bowel ulcers. Experience in one California country. Am J Gastroenterol 1968;50:449-55.

6 Grosfeld JL, Schiller M, Weinberger $M$, et al. Primary nonspecific ileal ulcers in children. Am J Dis Child 1970;120:447-50.

7 Campbell JR, Knapp RW. Small bowel ulceration associated with thiazide and potassium therapy. Review of 13 cases. Ann Surg 1966;163:291-6.

8 Bjarnason I, Hayllar J, MacPherson AJ, et al. Side effects of nonsteroidal antiinflammatory drugs on the small and large intestine in humans. Gastroenterology 1993;104:1832-47

9 Morris A. Nonsteroidal anti-inflammatory drug enteropathy. Gastrointest Endosc Clin N Am 1999;9:125-33.

10 Modigliani R, Poitras P, Galian A, et al. Chronic non-specific ulcerative duodenojejunoileitis. Report of four cases. Gut 1979;20:318-28.

11 Robertson DA, Dixon MF, Scott BB, et al. Small intestinal ulceration. Diagnostic difficulties in relation to coeliac disease. Gut 1983;24:565-74. 
12 Perlemuter G, Guillevin L, Legman P, et al. Cryptogenic multifocal ulcerous stenosing enteritis. An atypical type of vasculitis or a disease mimicking vasculitis? Gut 2001;48:333-8.

13 Okabe H, Sakimura M. Nonspecific multiple ulcer of the small intestine. Stomach and Intestine 1968;3:1539-49.

14 Sakimura M. Clinical study on "nonspecific multiple ulcers of the small intestine". Fukuoka lgaku Zasshi 1970;61:318-40.

15 Yao T, Fuchigami T, Sakimura M, et al. Proposal of a classification for small intestinal ulcers with a special reference to nonspecific multiple ulcers of the small intestine. Stomach and Intestine 1972;7:1615-20.

16 Wechsler B, Davachi F, Mizushima Y, et al. Criteria for diagnosis of Behcet's disease. International study group for Behcet's disease. Lancet 1990;335:1078-80.

17 Yao T, Matsui T, Hiwatashi N. Crohn's disease in Japan. Diagnostic criteria and epidemiology. Dis Colon Rectum 2000;43:S85-93.

18 Biarnason I, Price AB, Zanelli G, et al. Clinicopathological features of nonsteroidal antiinflammatory drug-induced small intestinal strictures. Gastroenterology 1988;94:1070-4.

19 Fellows IW, Clarke JM, Roberts PF. Non-steroidal anti-inflammatory druginduced jejunal and colonic diaphragm disease: a report of two cases. Gut 1992;30:1424-6.

20 Lang J, Price $A B$, Levi $A J$, et al. Diaphragm disease: pathology of disease of the small intestine induced by non-steroidal anti-inflammatory drugs. J Clin Pathol 1988;41:516-26.
21 Robinson $\mathrm{MH}$, Wheatley $\mathrm{T}$, Leach $\mathrm{H}$. Nonsteroidal antiinflammatory druginduced colonic stricture. An unusual cause of large bowel obstruction and perforation. Dig Dis Sci 1995;40:315-19.

22 Cortina G, Wren S, Armstrong B, et al. Clinical and pathological overlap in nonsteroidal anti-inflammatory drug-related small bowel diaphragm disease and the neuromuscular and vascular hamartoma of the small bowel. Am J Surg Pathol 1999;23:1414-17.

23 Santolaria S, Cabezari R, Ortego J, et al. Diaphragm disease of the small bowel. A case without apparent nonsteroidal antiinflammatory drug use. J Clin Gastroenterol 2001;32:344-6.

24 Brown CH, Akin N. Stenosing small-intestinal ulcers. Report of eleven cases. Cleve Clin Quart 1966;34:85-93.

25 Davies DR, Brightmore T. Idiopathic and drug-induced ulceration of the small intestine. Br J Surg 1970:57:134-9.

26 Lawrason FD, Alpert E, Mohr FL. Ulcerative-obstructive lesions of small intestine. JAMA 1965;191:641-4.

27 Biagi F, Lorenzini P, Corazza GR. Literature review on the clinical relationship between ulcerative jejunoileitis, coeliac disease, and enteropathy associated T-cell lymphoma. Scand J Gastroenterol 2000;35:785-90.

28 Chagnon JP, Devars du Mayne JF, Marche C, et al. Multifocal cryptogenic stenosing enteritis. An autonomous entity? Gastroenterol Clin Biol 1984;8:808-13.

29 Perlemuter G, Chaussade S, Soubrane O, et al. Multifocal stenosing ulcerations of the small intestine revealing vasculitis associated with $\mathrm{C} 2$ deficiency. Gastroenterology 1996;110:1628-32. 